\title{
Numerical Study of Hypersonic Wind Tunnel Experiments for Mars Entry Aeroshells
}

\author{
Hicham Alkandry ${ }^{*}$ and Iain D. Boyd ${ }^{\dagger}$ \\ Department of Aerospace Engineering, University of Michigan, Ann Arbor, MI, 48109 \\ Erin M. Reed ${ }^{\ddagger}$ and James C. McDaniel ${ }^{\S}$ \\ Department of Aerospace and Mechanical Engineering, University of Virginia, Charlottesville, VA, 22903
}

The combination of landing future high mass systems with small landing footprints on Mars may require the use of both propulsive deceleration (PD) and reaction control system (RCS) thrusters. However, the interactions between these jets and the supersonic or hypersonic freestream involve complex flow phenomena that are still not well understood. This paper describes numerical and experimental techniques that are used in an effort to develop physically accurate methods to compute these complex flow interactions. The paper also presents a numerical parametric study that is conducted using the computational fluid dynamics (CFD) code LeMANS. This study examines the effects of low temperature and density values and radially nonuniform freestream conditions in the hypersonic wind tunnel facility using an aeroshell based on the Mars Science Laboratory in Mach 12 flow of nitrogen gas with PD and RCS jets off. It is shown that although the Blottner and the Sutherland models compute different values of viscosity at low temperatures, the flowfield and surface properties predicted by LeMANS using these two models are in very close agreement. The study also shows that thermal nonequilibrium effects are negligible. The radial freestream nonuniformities, however, have considerable effects on the flowfield and surface properties. The nonuniform conditions change the temperature and density distributions in most of the computational domain, widen the bow shock around the aeroshell, increase continuum breakdown regions, and decrease the drag coefficient of the capsule compared to the uniform conditions. Finally, the paper presents qualitative experimental comparisons with the computed results which show overall good agreement.

\section{Nomenclature}

$\begin{array}{ll}C_{f} & =\text { Shear Stress Coefficient } \\ C_{p} & =\text { Pressure Coefficient } \\ K n & =\text { Global Knudsen Number } \\ K n_{G L L} & =\text { Gradient Length Local Knudsen Number } \\ M & =\text { Mach Number } \\ T & =\text { Temperature, } \mathrm{K} \\ U & =\text { Speed, } \mathrm{m} / \mathrm{s} \\ \alpha & =\text { Angle of Attack, degrees } \\ \lambda & =\text { Mean Free Path, } \mathrm{m} \\ \mu & =\text { Viscosity, } \mathrm{N} \cdot \mathrm{s} / \mathrm{m}^{2} \\ \rho & =\text { Density, } \mathrm{kg} / \mathrm{m}^{3}\end{array}$

\footnotetext{
${ }^{*}$ Graduate Student, Student Member AIAA.

${ }^{\dagger}$ Professor, Associate Fellow AIAA.

* Graduate Student, Student Member AIAA.

${ }^{\S}$ Professor, Senior Member AIAA.
} 


\section{Introduction}

$\mathrm{T}$ HE Mars Science Laboratory (MSL) spacecraft, scheduled to launch in the fall of 2011, will set new achievements in Mars atmospheric entry. The MSL will land a large rover with a footprint smaller than $10 \mathrm{~km}$ at altitudes much higher than has been previously attempted. ${ }^{1}$ It will have a greatly improved landing accuracy by flying at an angle of attack as large as -17 degrees and using a guidance system to bank the vehicle to rotate the lift vector in the desired direction. Reaction control system (RCS) thrusters will provide the torque to drive a coordinated bank. These RCS thrusters, located on the backshell, can cause jet plume interference effects which can generate large localized heating loads and can also interact with the rarefied freestream. The MSL spacecraft will also push the limits of conventional aerodynamic decelerators (i.e. blunt body and parachutes) due to its very high mass. At an estimated landing mass greater than $1700 \mathrm{~kg}$, the MSL will far exceed the entry mass for any previous Mars entry system (e.g. Viking). Therefore, future heavier Mars entry missions may require an additional propulsive deceleration (PD) component. However, the use of propulsive retrorockets has several issues primarily due to the significant limitations that exist in the current experimental database. ${ }^{2}$

The combination of landing future higher mass systems with greater accuracy on Mars may require the use of both PD and RCS thrusters. However, the interactions between these jets and the supersonic freestream involve complex flow phenomena that are still not well understood. In a combined study, researchers at the University of Michigan and the University of Virginia are using numerical and experimental methods to study the complex flow interactions that are generated in the use of PD and RCS jets. The research objectives are four-fold. The first objective is to design, build and install MSL models with PD and RCS jets in a hypersonic flow facility at the University of Virginia. The second objective is to use the planar laser-induced iodine fluorescence (PLIIF) technique to measure the flowfield interactions between these PD jets, RCS jets, the aeroshell and the supersonic freestream. The third objective is to develop effective procedures for physically accurate and numerically efficient computation of the complex flow interactions that are generated in the use of these PD and RCS jets for vehicle deceleration and control during Mars entry. The fourth and final objective is to validate the computational method by direct quantitative comparisons with the experimental measurements.

This paper will describe the numerical and experimental approaches that are used to achieve these objectives. It will also present a numerical parametric study that is carried out using the base model (i.e. PD and RCS jets off) to understand the effects of several parameters in the experimental facility in an effort to fulfill the third and fourth research objectives. These numerical results are presented in three parts. First, the effects of low freestream temperatures and densities in the experimental setting are examined using axisymmetric simulations. Second, the effects of radially nonuniform freestream conditions in the experimental facility are studied using axisymmetric simulations. Finally, qualitative comparisons with experimental data are presented.

\section{Technical Approach}

\section{A. Experimental Technique}

Experimental measurements are obtained using the planar laser-induced iodine fluorescence (PLIIF) technique at a hypersonic wind tunnel at the University of Virginia. The PLIIF technique is a non-intrusive, optical method for measurements in hypersonic, rarefied flows. ${ }^{3}$ The technique has been used for both qualitative and quantitative measurements. PLIIF involves seeding iodine into a flowfield and exciting the molecules to a higher energy with an argon ion laser. The laser beam is turned into a thin laser sheet and passed through the flowfield of interest. The resulting fluorescence is imaged at 90 degrees using a cooled scientific-grade charge-coupled device (CCD) camera. Measurements of the absorption spectrum are made as the laser is tuned in frequency. By fitting the measured absorption spectra at every point in the flowfield, the velocity, temperature and injectant mole fraction can be deduced.

The hypersonic flow facility at the University of Virginia is capable of providing Mach numbers and Knudsen numbers up to 16 and 1, respectively. Hypersonic flow from an underexpanded jet is produced by the expansion of $\mathrm{I}_{2}$-seeded $\mathrm{N}_{2}$ gas across a thin circular orifice of diameter $D=2 \mathrm{~mm}$ into a continuously evacuated vacuum chamber. Figure 1 shows a schematic of the experimental setup in the hypersonic flow facility. Figure $2^{4}$ shows calculated Mach number and Knudsen number $(K n)$ variations inside the freejet facility. These contours show the barrel shock that develops at the entrance of the test section and terminates at the Mach disk. Models are placed in the underexpanded jet core for testing at hypersonic conditions. The freestream Mach number and flow properties can be changed by adjusting the distance of the test model to the orifice. 


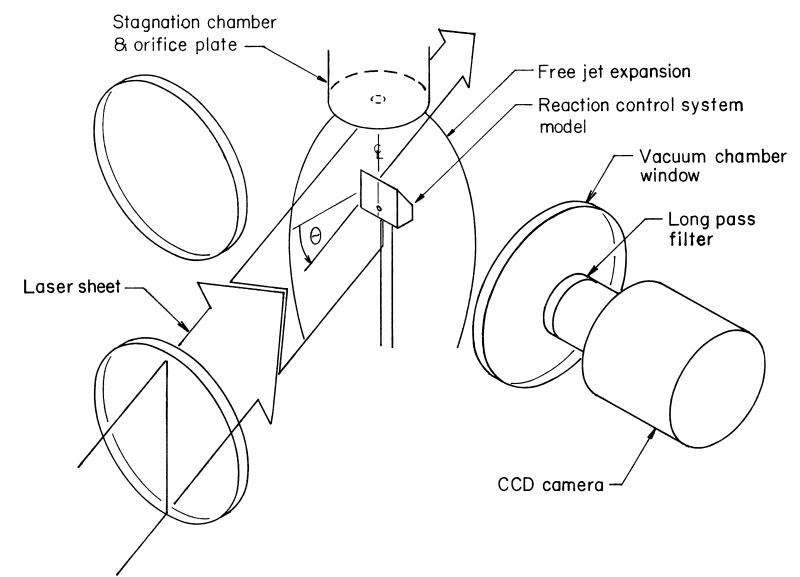

Figure 1. Schematic of the experimental setup.

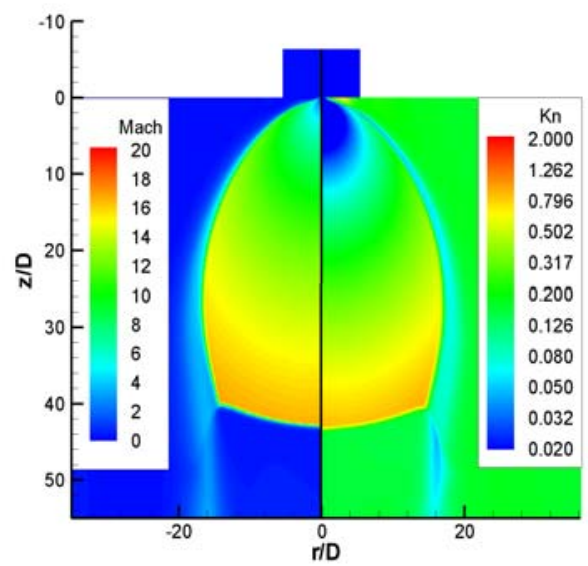

Figure 2. Calculated Mach number and Knudsen number variations in the test section.

\section{B. Numerical Method}

Numerical simulations are performed using the computational fluid dynamics (CFD) code LeMANS, 5,6 developed at the University of Michigan for simulating hypersonic reacting flows. This general purpose, threedimensional, parallel code solves the laminar Navier-Stokes equations on unstructured computational grids including thermo-chemical nonequilibrium effects with second-order accuracy. In LeMANS, the flow is modeled assuming that the continuum approximation is valid and that the fluid can be weakly ionized. Furthermore, it is assumed that the translational and rotational energy modes of all species can be described by two different temperatures $T$ and $T_{r}$, respectively, while the vibrational energy mode and electron energy of all species can be described by a single temperature $T_{v}{ }^{7}$ The electronic energy of atoms and molecules is neglected in the current version of the code due to the relatively small temperatures achieved in the hypersonic flows of interest.

The finite-volume method applied to unstructured grids is used to solve the set of differential equations. LeMANS can simulate two-dimensional/axisymmetric flows using any mixture of quadrilateral and triangular mesh cells, and three-dimensional flows using any mixture of hexahedra, tetrahedra, prisms, and pyramids. It employs a modified Steger-Warming Flux Vector Splitting scheme to discretize the inviscid fluxes across cell faces, which is less dissipative and produces better results in boundary layers compared to the original scheme. Viscous terms are computed using cell center and node values. Time marching is performed using either a point implicit or a line implicit method. LeMANS is parallelized using METIS $^{8}$ to partition the computational mesh between processors and MPI to communicate information between processors.

In LeMANS, the mixture transport properties are calculated using two different models. Both models use Wilke's semi-empirical mixing ${ }^{9}$ with species thermal conductivities determined using Eucken's relation. ${ }^{10}$ The first option computes the species viscosities using the Blottner model ${ }^{11}$, which is a set of curve fits given by Eq. (1)

$$
\mu=0.1 \exp [(A \ln T+B) \ln T+C]
$$

where $\mu$ is the viscosity, $T$ is the translational temperature, and $A, B$, and $C$ are constants equal to $0.0268,0.318$, and -11.3 , respectively, for $\mathrm{N}_{2}$. The second option uses the Sutherland model, which is a simple algebraic equation given by Eq. $(2)^{12}$

$$
\mu=\mu_{0}\left(\frac{T}{T_{0}}\right)^{3 / 2}\left(\frac{T_{0}+S}{T+S}\right)
$$

where $\mu_{0}, T_{0}$ and $S$ are constants equal to $1.663 \times 10^{-5} \mathrm{~N} \cdot \mathrm{s} / \mathrm{m}^{2}, 273 \mathrm{~K}$, and $107 \mathrm{~K}$, respectively, for $\mathrm{N}_{2}$. Figure 3 shows a comparison between the Blottner curve fit data and the Sutherland model for $\mathrm{N}_{2}$ at a range of temperatures that is of interest to this study. The figure also includes experimental measurements performed at the National Institute of Standards and Technology (NIST) ${ }^{13}$. As can be seen from the figure, the Sutherland model agrees better with the experimental data, particularly at low temperatures, than the Blottner curve fit data. 


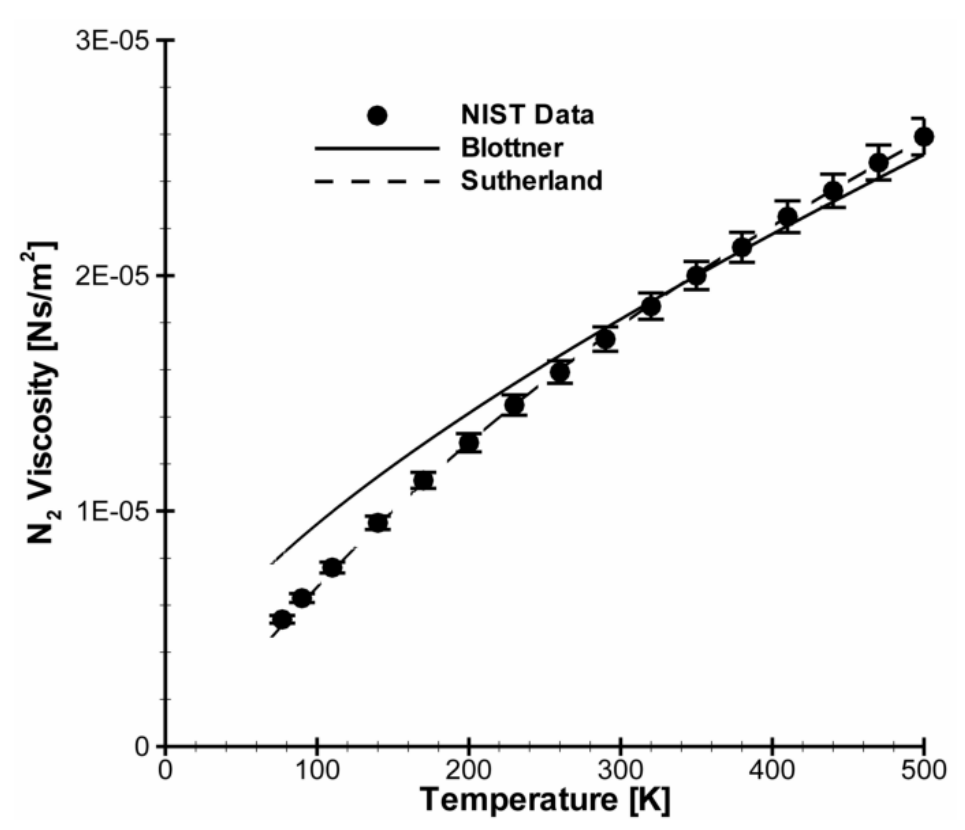

Figure 3. Viscosity of $\mathbf{N}_{2}$ as a function of temperature.

\section{Numerical Setup}

The geometry of the test model used in this study is presented in Fig. 4. The model is a scaled down version of the MSL aeroshell geometry. The model's diameter is $20 \mathrm{~mm}$ and the nose radius and angle are $5 \mathrm{~mm}$ and $70 \mathrm{deg}$, respectively. Section $A A$ represents the geometry used in the forebody axisymmetric simulations.

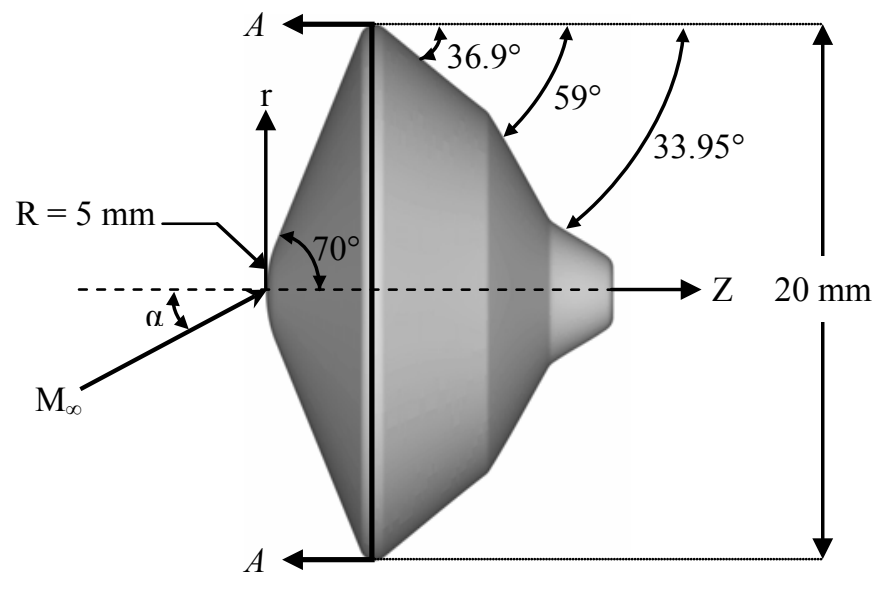

Figure 4. Model geometry.

The freestream conditions and wall temperature for the numerical simulations are provided in Table 1 . The freestream Mach number is 12 and is chosen to match the experimental settings at an angle of attack of 0 degrees. The freestream temperature, density, and speed are $10 \mathrm{~K}, 4.2 \times 10^{-4} \mathrm{~kg} / \mathrm{m}^{3}$, and $776 \mathrm{~m} / \mathrm{s}$, respectively, and are computed using isentropic gas relations for $\mathrm{N}_{2}$ at Mach 12. The stagnation temperature and pressure in the wind tunnel are $300 \mathrm{~K}$ and $182.7 \mathrm{kPa}$, respectively. At these conditions, chemical nonequilibrium effects can be neglected. Thermal equilibrium is also assumed, except for one simulation where possible nonequilibrium effects, particularly for the rotational mode, are examined. Although the $\mathrm{N}_{2}$ gas in the experimental facility is seeded with $\mathrm{I}_{2}$ with a seeding ratio of approximately $200 \mathrm{I}_{2}$ molecules per $10^{6} \mathrm{~N}_{2}$ molecules, Padilla ${ }^{14}$ showed that the differences in flowfield properties between $\mathrm{I}_{2}$-seeded and pure $\mathrm{N}_{2}$ gas are overall small, with slight discrepancies in velocity components in the boundary layer and the shock. Therefore, pure $\mathrm{N}_{2}$ instead of $\mathrm{I}_{2}$-seeded gas is considered in the 
numerical simulations. The freestream global Knudsen number, given by Eq. (3) and equal to $7.7 \times 10^{-3}$, indicates that the flow is in the near-continuum regime

$$
K n=\frac{\lambda}{L}
$$

where $\lambda$ is the mean free path and $L$ is a characteristic length scale equal to the model's diameter.

Table 1. Freestream conditions and wall temperature for the numerical simulations.

\begin{tabular}{|c|c|c|c|c|c|}
\hline $\begin{array}{c}\text { Mach Number, } \\
M_{\infty}\end{array}$ & $\begin{array}{c}\text { Temperature, } \\
T_{\infty}[\mathrm{K}]\end{array}$ & $\begin{array}{c}\text { Density, } \\
\rho_{\infty}\left[\mathrm{kg} / \mathrm{m}^{3}\right]\end{array}$ & $\begin{array}{c}\text { Speed, } \\
U_{\infty}[\mathrm{m} / \mathrm{s}]\end{array}$ & $\begin{array}{c}\text { Knudsen } \\
\text { Number, } \mathrm{Kn}_{\infty}\end{array}$ & $\begin{array}{c}\text { Wall Temperature, } \\
T_{\text {wall }}[\mathrm{K}]\end{array}$ \\
\hline \hline 12 & 10 & $4.2 \times 10^{-4}$ & 776 & $7.7 \times 10^{-3}$ & 300 \\
\hline
\end{tabular}

In order to better simulate the flow in the experimental facility, radially nonuniform conditions based on the supersonic freejet relations of Ashkenas and Sherman ${ }^{15}$ are used as boundary conditions to LeMANS. The Mach number at a distance $x$ along the centerline of the freejet is given by Eq. (4)

$$
M=A\left(\frac{x-x_{0}}{D}\right)^{\gamma-1}-\frac{1}{2}\left(\frac{\gamma+1}{\gamma-1}\right)\left[A\left(\frac{x-x_{0}}{D}\right)^{\gamma-1}\right]^{-1}
$$

where $D$ is the diameter of the jet orifice, and $A$ and $x_{0} / \mathrm{D}$ are constants based on the ratio of specific heats $\gamma$ and are equal to 3.65 and 0.40 , respectively, for $\mathrm{N}_{2}$ gas. All other fluid properties can be computed along the jet axis using the Mach number defined by Eq. (4), the stagnation conditions and the isentropic relations. The density distribution as a function of the streamline angle $\theta$ with respect to the jet axis is given by Eq. (5)

$$
\frac{\rho(\theta)}{\rho(0)}=\cos ^{2}\left(\frac{\pi \theta}{2 \Phi}\right)
$$

where $\Phi$ is also a constant based on the ratio of specific heats $\gamma$ and is equal to 1.662 for $\mathrm{N}_{2}$.

Table 2 presents information about the computational meshes used for the simulations. These meshes are generated using the commercial software GAMBIT ${ }^{16}$. A grid-convergence study is performed to ensure that all of the solutions are grid-independent. The grids are structured with quadrilateral elements. These elements are chosen because the numerical results are very sensitive to the alignment of the shock wave with the grid. Cell stretching is also used to cluster nodes near the surface of the model. Some of the full body, axisymmetric simulations require a modified computational domain to accurately predict the bow shock location behind the aeroshell in the wake region. Figure 5 shows the meshes for the axisymmetric simulations. A point implicit method is used for time marching, which is then switched to a line implicit method after a few thousand iterations to accelerate convergence. This switch between the two methods also insures that the solution does not diverge. The gradient length local Knudsen number, given by Eq. (6), is used as a parameter to determine continuum breakdown ${ }^{17}$

$$
K n_{G L L}=\frac{\lambda}{Q}\left|\frac{d Q}{d l}\right|
$$

where $Q$ is a variable of interest (e.g. temperature, density, pressure), and the derivative is taken in the direction of maximum gradient. Generally, for $K n_{G L L}$ values above 0.05 , continuum breakdown occurs and the Navier-Stokes equations become physically inaccurate.

Table 2. Computational grids.

\begin{tabular}{|l|c|c|c|}
\hline \multicolumn{1}{|c|}{ Case } & $\begin{array}{c}\text { Total Number of } \\
\text { Cells }\end{array}$ & $\begin{array}{c}\text { Number of Cells in } \\
\text { Normal Direction }\end{array}$ & $\begin{array}{c}\text { Number of Cells on } \\
\text { the Surface }\end{array}$ \\
\hline \hline Axisymmetric - Forebody & 29,300 & 407 & 72 \\
\hline Axisymmetric - Full Body & 188,730 & 405 & 271 \\
\hline Axisymmetric - Modified Full Body & 188,730 & 405 & 271 \\
\hline
\end{tabular}




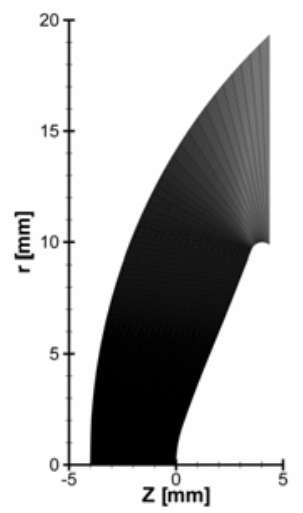

(a) Forebody

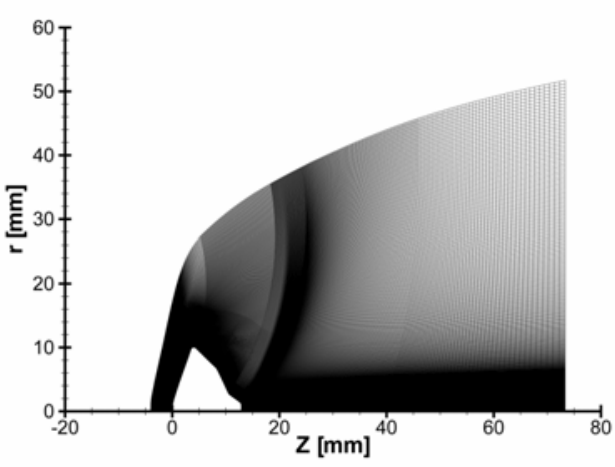

(b) Full Body

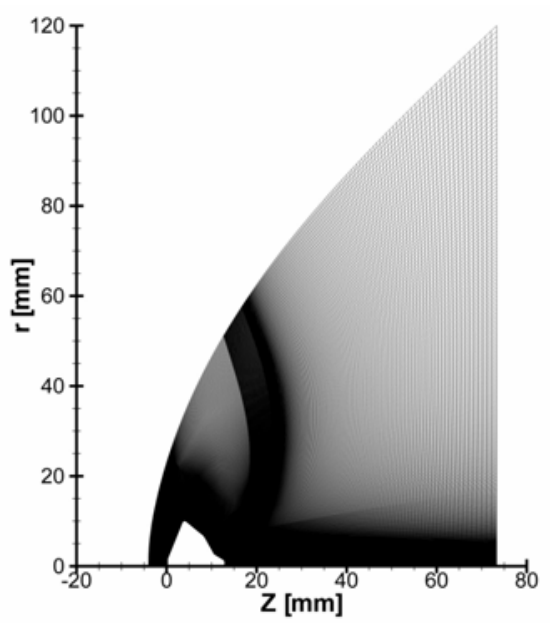

(c) Modified Full Body

Figure 5. Final computational grids used in the numerical simulations.

\section{Results}

The purpose of this study is to determine the effects of low temperature and density values and radially nonuniform conditions in the experimental facility on the flowfield and surface properties around an MSL-based model with PD and RCS jets off using the CFD code LeMANS. The numerical results are also used in qualitative experimental comparisons to assess the numerical method, which will be used to simulate the interactions between PD jets, RCS jets, the aeroshell, and the hypersonic freestream in future work. The flowfield properties that are used in studying the effects of these parameters are temperature, density, and gradient length local Knudsen number. The surface properties are presented as non-dimensionalized coefficients defined by Eqs. (7) and (8)

$$
\begin{aligned}
& C_{p}=\frac{p}{\frac{1}{2} \rho_{\infty} U_{\infty}^{2}} \\
& C_{f}=\frac{\tau}{\frac{1}{2} \rho_{\infty} U_{\infty}^{2}}
\end{aligned}
$$

where $p$ is the pressure and $\tau$ is the shear stress.

\section{A. Effects of Highly Expanded Freestream}

The low freestream temperature and density in the experimental facility may have several effects on the numerical solutions computed by LeMANS. First, as can be seen from Fig. 3, the viscosity values computed using the Blottner curve fit data and the Sutherland model are different, particularly at low temperatures. For example, at $80 \mathrm{~K}$, the dynamic viscosity for the Blottner curve fit data is $8.3 \times 10^{-6} \mathrm{~N} \cdot \mathrm{s} / \mathrm{m}^{2}$, whereas the value for the Sutherland model is $5.4 \times 10^{-6} \mathrm{~N} \cdot \mathrm{s} / \mathrm{m}^{2}$, which represents a 42 percent difference. Therefore, it is important to understand how this difference in viscosity between the two models affects the flowfield and surface properties. Second, at these low densities, thermal nonequilibrium effects, especially for the rotational mode, may become significant and would therefore need to be accounted for in the numerical simulations.

\section{Viscosity Model}

Figure 6 presents the results of the full body, axisymmetric simulations that are performed using the Sutherland model (top half) and the Blottner curve fit data (bottom half). The run time for each of these simulations is approximately 27 hours on 16 Intel Xeon X5355 processors. Figures 6(a), (b), and (c) show contours of temperature, density and $K n_{G L L}$, respectively, and Fig. 6(d) shows a plot of the non-dimensionalized surface properties. The temperature and density contours show the bow shock around the aeroshell. The $K n_{G L L}$ contour plot exposes continuum breakdown regions (values greater than 0.05 ) in the bow shock and also behind the aeroshell in the wake. Comparisons of the contour plots between the Sutherland model and the Blottner curve fit data show almost no differences between the two models, which suggests that flowfield properties are not sensitive to the change in 
viscosity values. This close agreement between the results for the Blottner curve fit data and the Sutherland model can be explained by the fact that the temperatures in the regions where the viscous effects are important (e.g. the bow shock, the boundary layer and the expansion fan), are in a range (200-300 K) where the two models compute similar viscosity values as shown in Fig. 3. Figure 6(d) is a plot of the surface properties for the two viscosity models. It can be seen from the plot that the pressure coefficient is almost constant over the forebody of the aeroshell, with a maximum value of 1.85 for both the Sutherland model and the Blottner curve fit data at the stagnation point. Near the aeroshell shoulder, the pressure coefficient drops sharply to almost a constant value of 0.03 for both models. It can also be seen from Fig. 6(d) that the shear stress coefficient increases along the forebody, until it reaches a maximum value of 0.326 for both the Sutherland model and the Blottner curve fit data at the aeroshell shoulder. The value of the shear stress coefficient then decreases until reaching the third shoulder of the aeroshell, where it starts increasing again before it sharply decreases to zero at the base of the capsule. This plot shows that the results for the Sutherland model and the Blottner curve fit data are almost identical, which suggests that the surface properties are also not sensitive to the viscosity changes. This agreement is also due to the similar viscosity values computed by the two models at temperature values close to the wall temperature ( $300 \mathrm{~K}$ ). Therefore, the Blottner curve fit data can be used for all other numerical simulations.

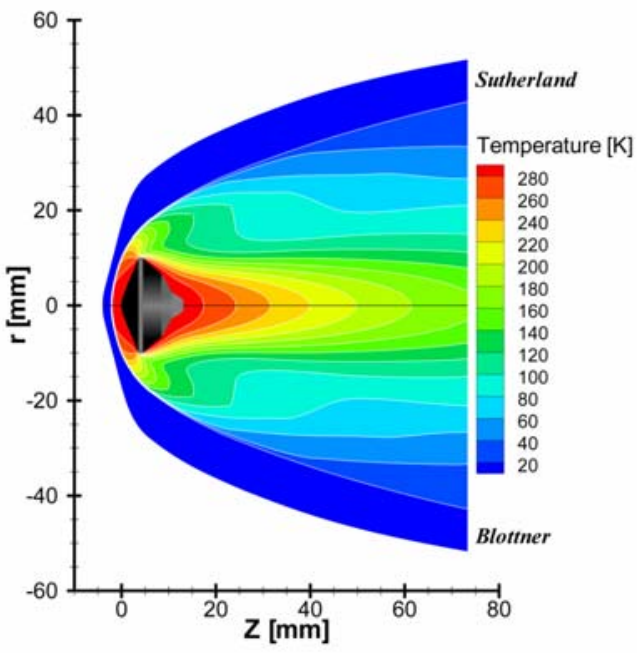

(a) Temperature

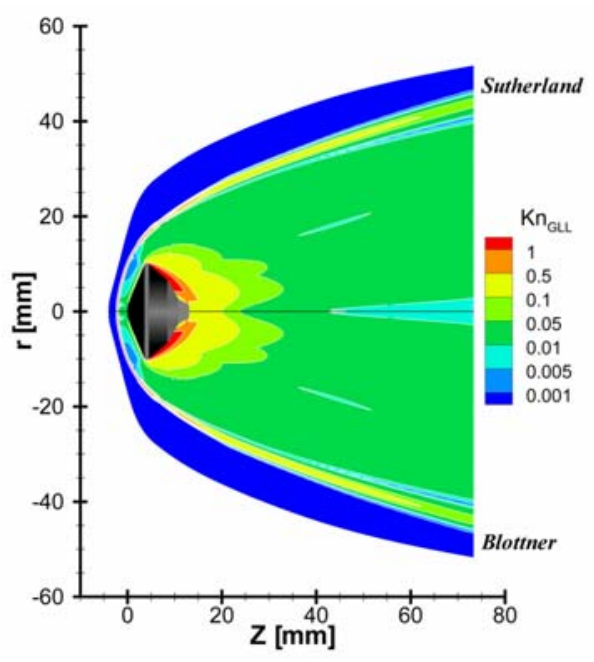

(c) $K n_{G L L}$

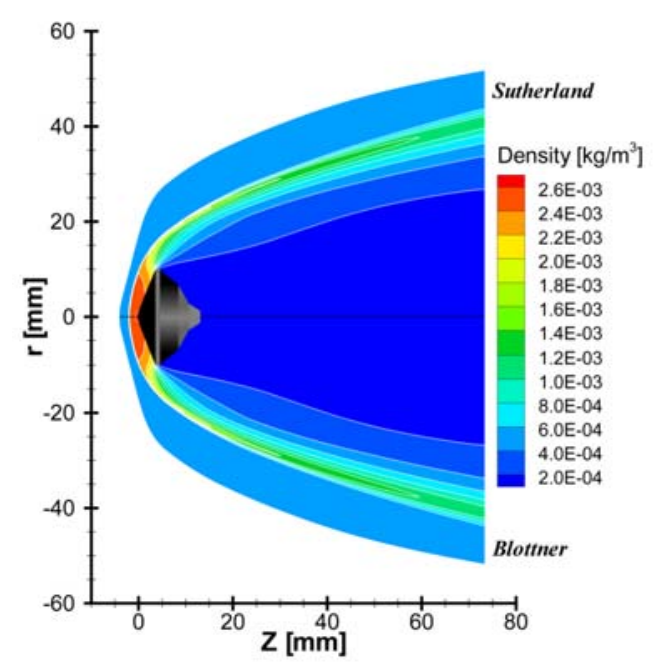

(b) Density

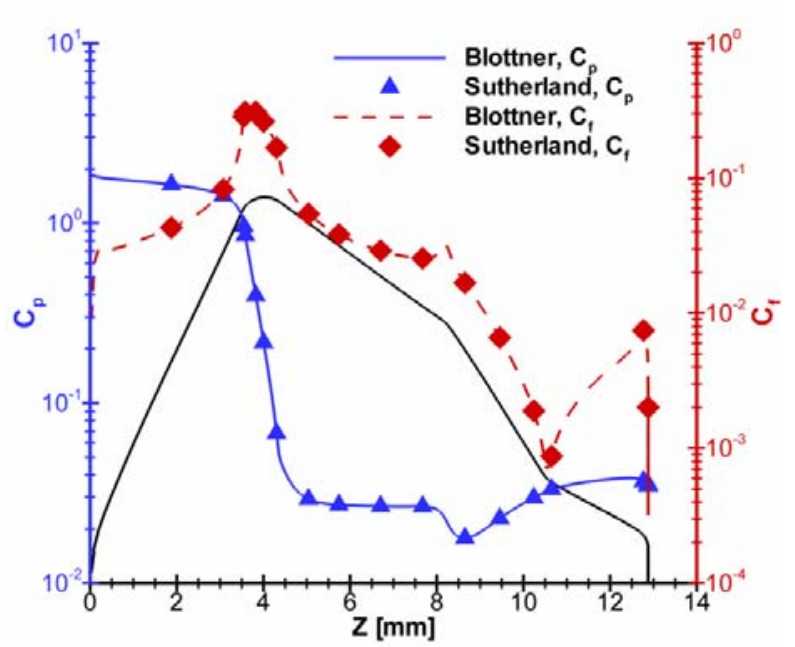

(d) Surface Properties

Figure 6. Comparison between Blottner curve fit data and the Sutherland model for full body, axisymmetric simulations at Mach 12. 


\section{Thermal Nonequilibrium}

Thermal nonequilibrium effects, particularly for the rotational mode, are evaluated using full body, axisymmetric simulations at Mach 12. Figure 7 shows contour plots for thermal nonequilibrium (top half) and equilibrium (bottom half) as well as a plot of the surface properties. The freestream and wall rotational temperature values are assumed to be $10 \mathrm{~K}$ and $300 \mathrm{~K}$, respectively. The vibrational temperature is assumed frozen at the stagnation value and therefore constant in the entire domain at $300 \mathrm{~K}$. The simulation run times for the thermal nonequilibrium and thermal equilibrium cases are approximately 42 and 27 hours, respectively, with each simulation using 16 Intel Xeon X5355 processors. The temperature, density and $K n_{G L L}$ contour plots in Figs. 7(a), (b) and (c) show very small differences between the two cases, with slight discrepancies in the temperature distribution in the wake. Figure 7(d) also shows almost no differences between the thermal nonequilibrium and thermal equilibrium cases in the pressure and shear stress coefficients. The reason for this close agreement between the two cases is that at these low temperatures, the rotational collision number and the mean collision time are both still small, which together give a fast relaxation time for the rotational mode. Therefore, it can be assumed that rotational nonequilibrium effects are negligible and a one-temperature model (translational mode) can be used for all other numerical simulations.

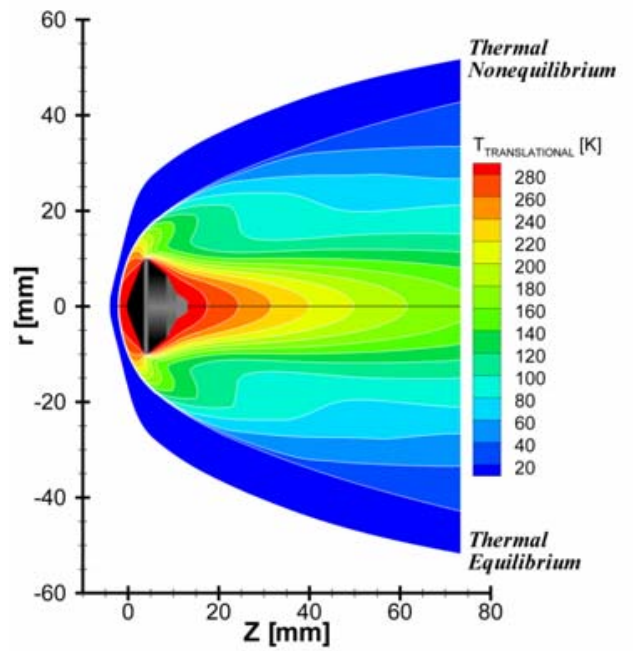

(a) Translational Temperature

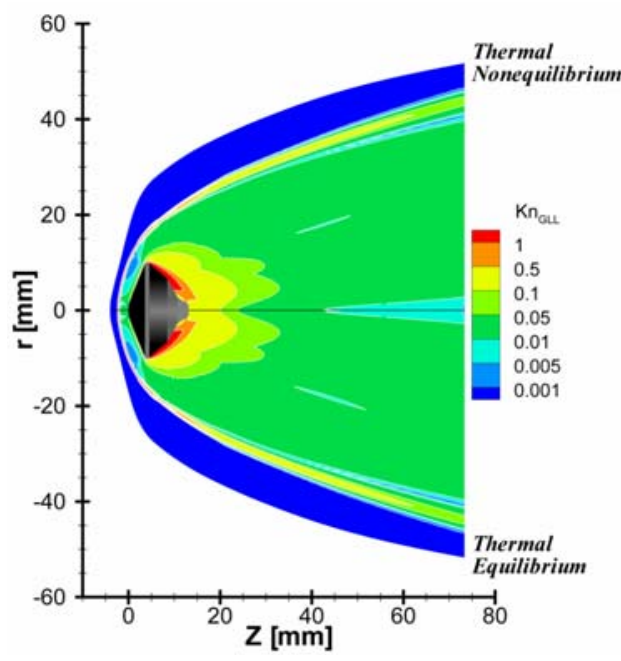

(c) $K n_{G L L}$

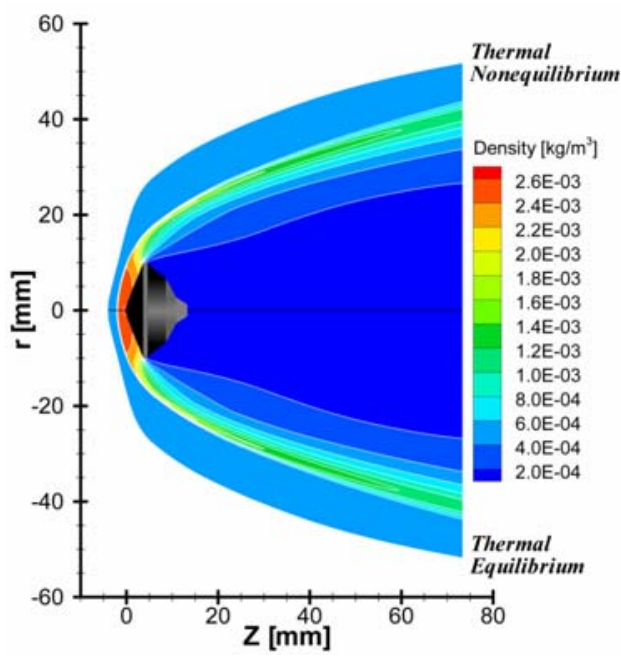

(b) Density

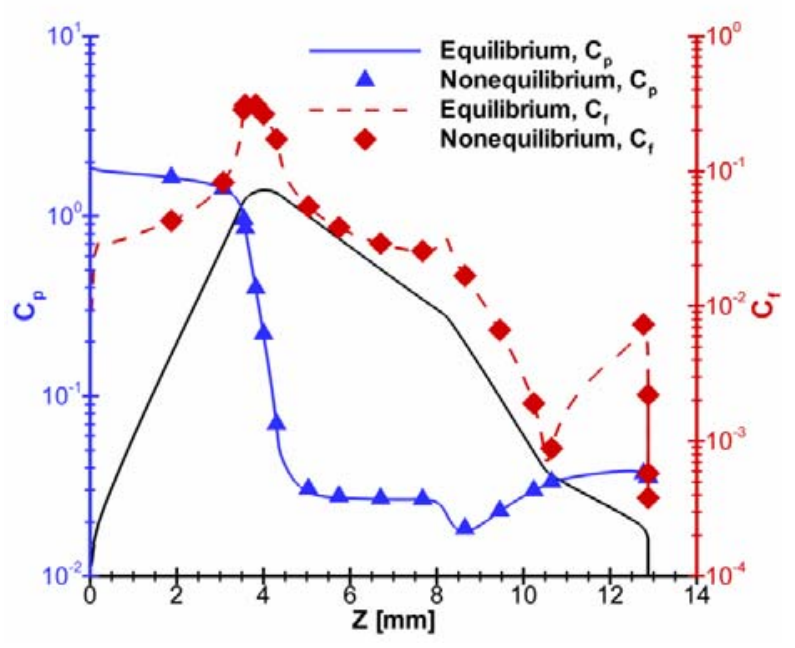

(d) Surface Properties

Figure 7. Comparison between thermal equilibrium and nonequilibrium for full body, axisymmetric simulations at Mach 12. 


\section{B. Effect of Radially Nonuniform Freestream Conditions}

The flow in the experimental facility at the University of Virginia is generated from an underexpanded jet. Therefore, it is important to understand the effects of the nonuniform freestream conditions on the flowfield and surface properties. This is achieved using forebody and full body axisymmetric simulations.

\section{Forebody, Axisymmetric Simulations}

The results of axisymmetric simulations using the forebody geometry at Mach 12 are presented in Fig. 8. The figure shows a comparison between the nonuniform (top half) and uniform (bottom half) freestream conditions using temperature, density and $K n_{G L L}$ contours. The run time for each of these two simulations is approximately 7 hours using 8 Intel Xeon X5355 processors. All of the contour plots in Fig. 8 show the bow shock in front of the model. The nonuniform freestream conditions, however, cause the shock to move closer to the nose of the aeroshell and away from its shoulder. The temperature and density contours show that the shock strength for the nonuniform conditions decreases at a faster rate due to the shock's interaction with a stronger expansion fan near the shoulder of the aeroshell compared to the uniform conditions. It can also be seen from Figs. 8(a) and (b) that there are significant variations in temperature and density distributions in the post-shock region between the two freestream conditions. Figure 8 (c) shows continuum breakdown regions (i.e. $K n_{G L L}>0.05$ ) in the shock, above the shoulder, and near the wall. It can also be seen from this contour plot that the nonuniform conditions cause an overall increase in $K n_{G L L}$ values compared to the results for the uniform conditions. The higher freestream $K n_{G L L}$ values for the nonuniform conditions are due to the gradients in flowfield properties associate with the underexpanded jet.

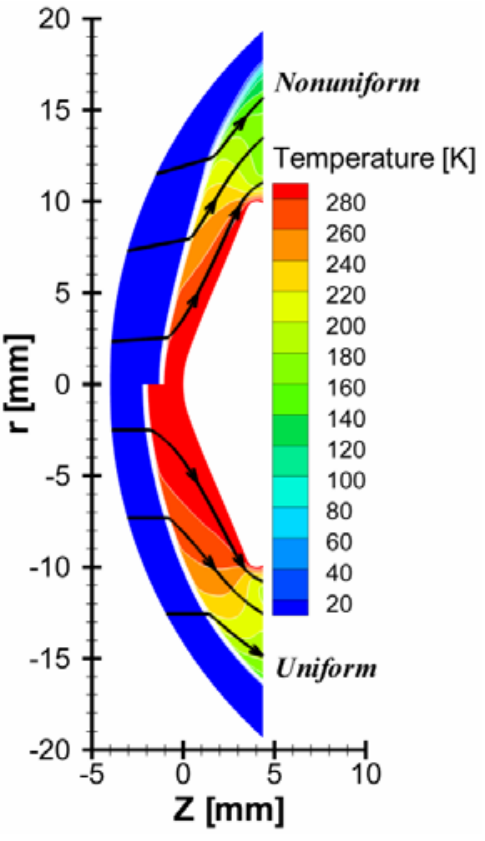

(a) Temperature

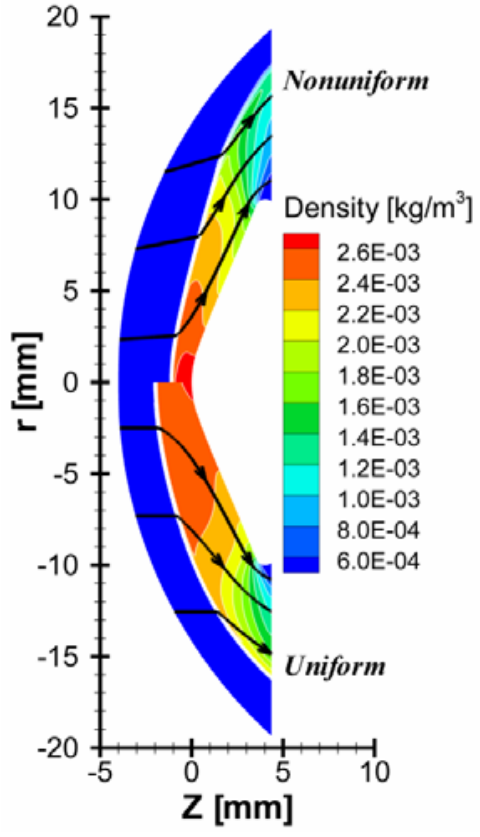

(b) Density

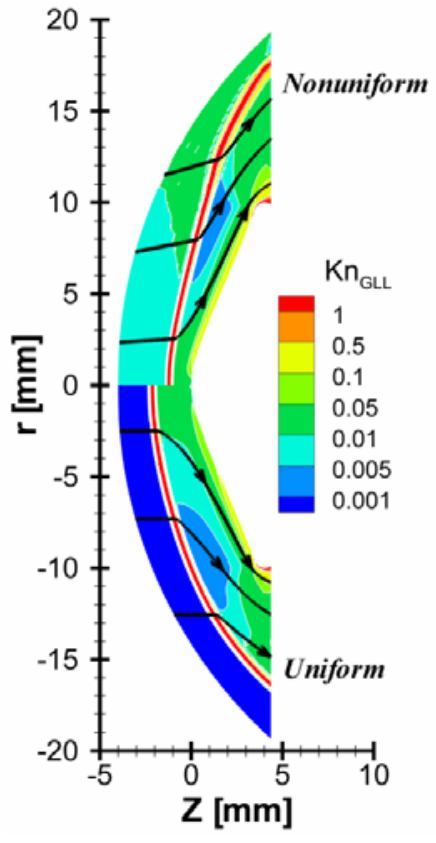

(c) $K n_{G L L}$

Figure 8. Comparison between uniform and nonuniform freestream conditions for forebody, axisymmetric simulations at Mach 12.

\section{Full Body, Axisymmetric Simulations}

The effects of the nonuniform freestream conditions in the experimental facility are also evaluated using full body and modified full body, axisymmetric simulations for the uniform and nonuniform conditions, respectively. The nonuniform freestream condition simulations use the mesh shown in Fig. 5(c) to accurately predict the bow shock location behind the aeroshell in the wake region. The run time for the uniform condition simulation is approximately 27 hours while the one for nonuniform condition simulation is about 34 hours, with each simulation running on 16 Intel Xeon X5355 processors. Figures 9(a), (b), and (c) show contour plots of temperature, density, and $K n_{G L L}$, respectively, for nonuniform (top half) and uniform (bottom half) freestream conditions. Similar to the results from the forebody, axisymmetric simulations, the contour plots show a wider bow shock due to the freestream nonuniformities. They also show that the shock strength decays faster in the region behind the aeroshell due to the interaction with a stronger expansion fan near the capsule's shoulder. The temperature and density 
variations in the post-shock and wake regions differ between the two freestream conditions, with overall lower temperature and density values for the nonuniform conditions, as can be seen from Figs. 9(a) and (b). The $K n_{G L L}$ contour plot, Fig. 9(c), shows continuum breakdown regions in the shock and behind the model in the wake. These regions are much larger for the nonuniform than for the uniform freestream conditions, particularly in the wake where the nonuniform conditions result in continuum breakdown in most of that region. Figure 9(d) compares the surface properties, $C_{p}$ and $C_{f}$, for the two freestream conditions. The plot shows that the stagnation pressure coefficient for the two freestream conditions are both equal to 1.85 and that the nonuniform conditions cause an overall decrease in pressure values over the entire surface of the aeroshell, especially in the aftbody. Figure 9(d) also shows differences in shear stress values, particularly near the base of the capsule, where the nonuniform conditions increase the shear stress values compared to the uniform conditions. As a result of these discrepancies in the surface properties, the drag coefficient of the aeroshell is also affected and decreases from 1.57 for the uniform to 1.47 for the nonuniform freestream conditions.

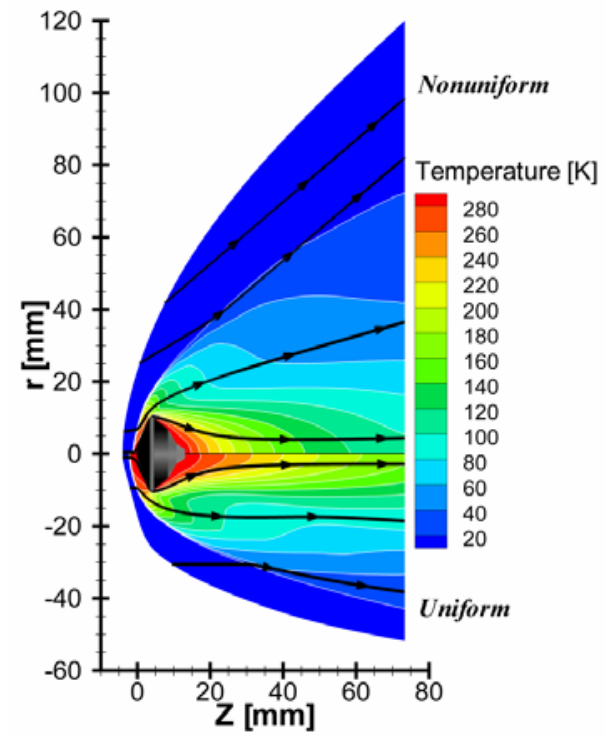

(a) Temperature

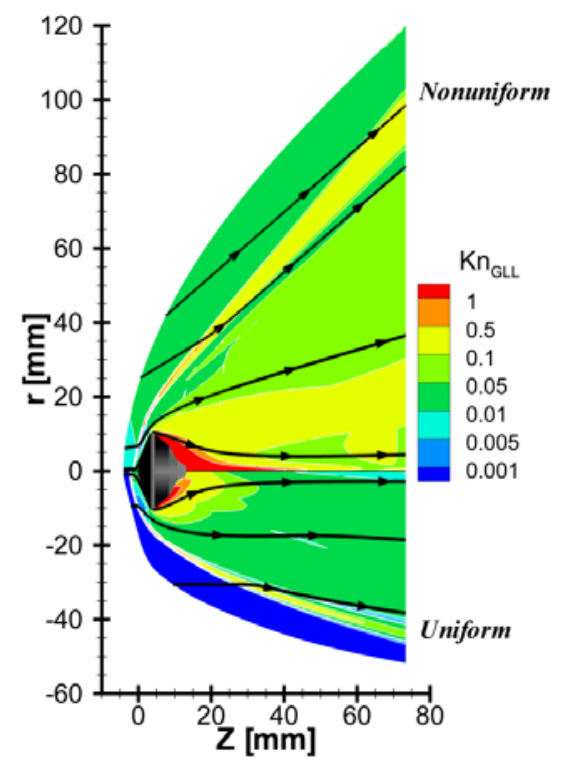

(c) $K n_{\text {GI.I. }}$

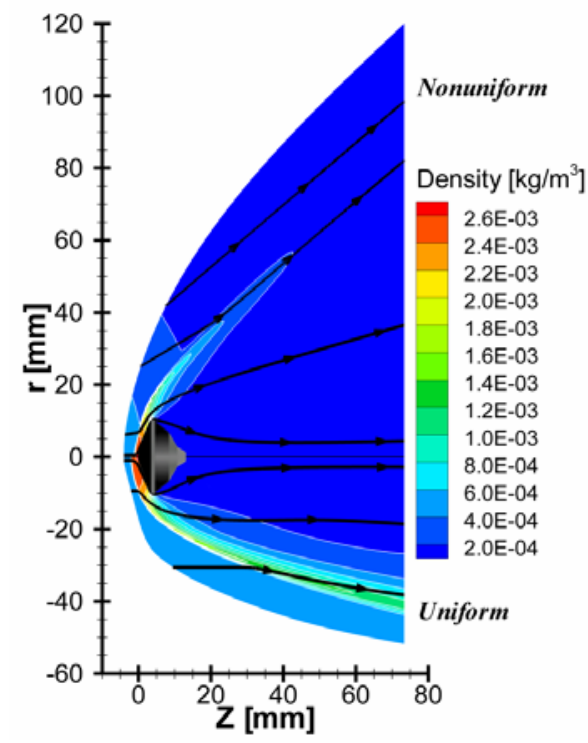

(b) Density

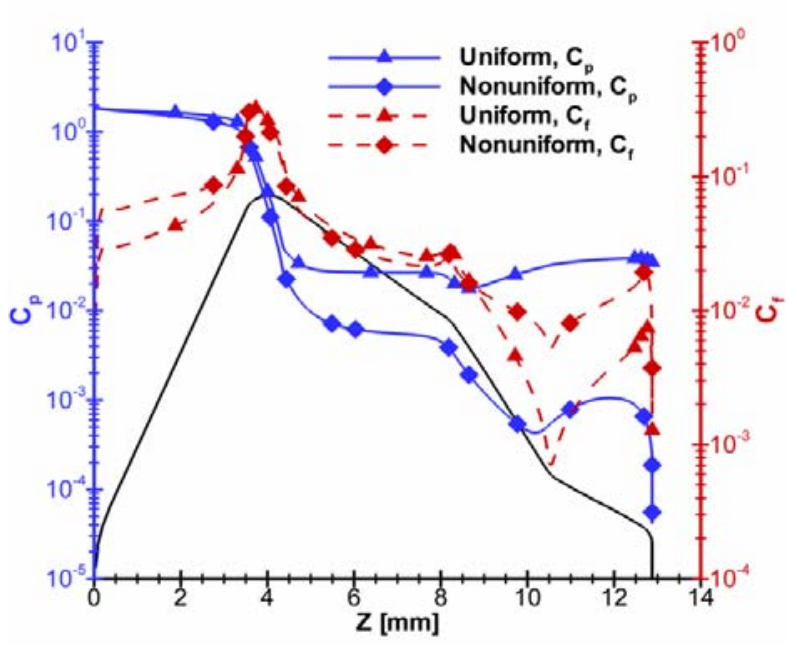

(d) Surface Properties

Figure 9. Comparison between uniform and nonuniform freestream conditions for full body, axisymmetric simulations at Mach 12. 


\section{Qualitative Experimental Comparison}

Comparisons with experimental visualizations from the University of Virginia are carried out to assess the numerical results. These experimental images are from plasma visualization, not planar laser-induced fluorescence, and are therefore path integrated and not spatially accurate. However, they can still clearly define the bow shock location, which is used as the comparison parameter. Figure 10 compares the numerical results for full body, axisymmetric simulations using uniform and nonuniform freestream conditions with experimental visualization at Mach 12. The light regions in the experimental visualizations represent areas with relatively high density values. The numerical results represent a density contour level with a value of $4.9 \times 10^{-4} \mathrm{~kg} / \mathrm{m}^{3}$, which is slightly larger than the freestream density value given in Table 1 . As can be seen from the figure, there is good agreement between the numerical results and the experimental visualizations, especially for the nonuniform freestream conditions where the shock locations are almost identical.

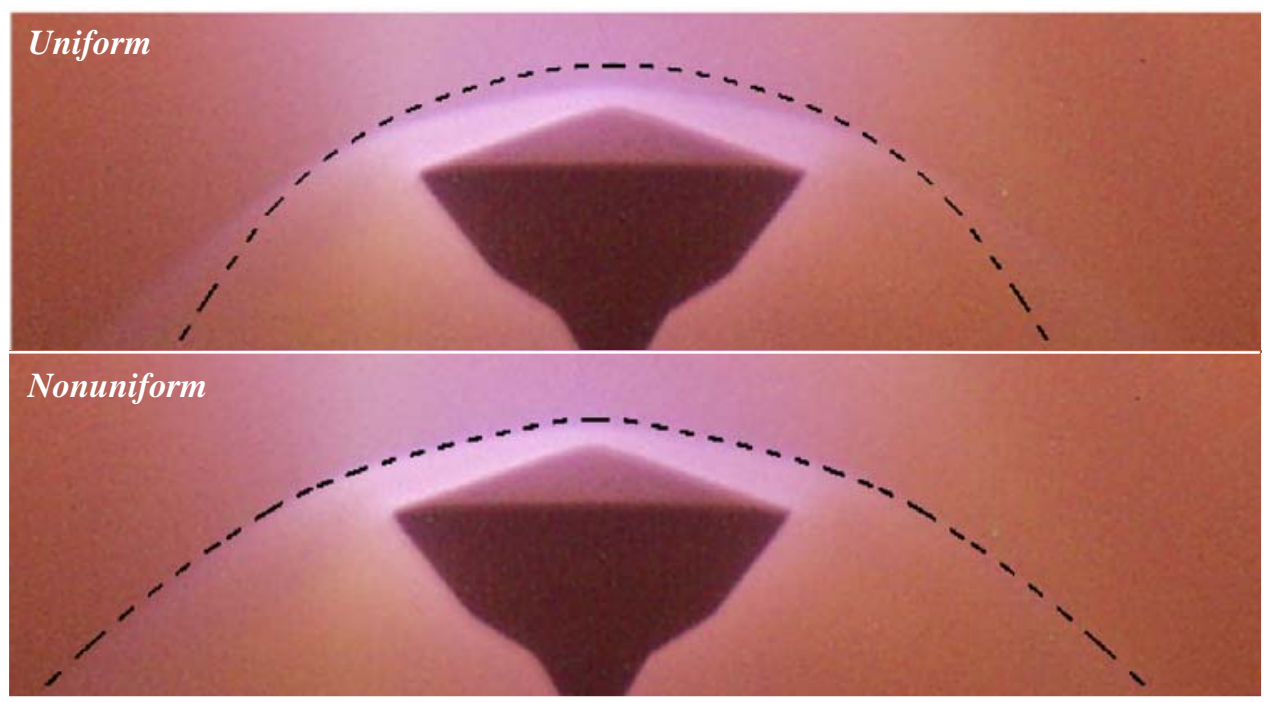

Figure 10. Comparison of bow shock location between numerical results and experimental visualization at Mach 12 and $\alpha=0^{\circ}$.

\section{Conclusion}

The purpose of this study was to determine the effects of low temperature and density values and radially nonuniform conditions in the hypersonic wind tunnel facility at the University of Virginia. Using the CFD code LeMANS, the effects on the flowfield and surface properties around an MSL-based aeroshell were evaluated at Mach 12 flow of nitrogen gas. The first part of this study focused on understanding viscosity and thermal nonequilibrium effects on temperature, density, continuum breakdown and surface pressure and shear stress. The results from axisymmetric simulations showed that although the viscosity was affected by the model used to evaluate it, particularly at low temperatures as experienced in the hypersonic flow facility, it had almost no effect on the flowfield and surface properties. It was also found that thermal nonequilibrium effects were negligible and the flow can be modeled using a one-temperature model. The second part of this study aimed to identify the effects of the radially nonuniform freestream conditions in the experimental facility on the same set of flowfield and surface properties. Axisymmetric simulation results showed that the nonuniform conditions widened the bow shock, changed temperature and density distributions over most of the computational domain, and caused larger continuum breakdown regions, particularly in the wake, when compared to the results for uniform freestream conditions. The nonuniform conditions also increased the values of the shear stress along the forebody and near the base of the aeroshell surface and decreased the overall surface pressure values. As a result, there was a 6.4 percent decrease in drag coefficient due to the nonuniform freestream conditions. Qualitative comparisons with experimental visualizations at 0 degrees angle of attack showed very good agreement in the bow shock location, especially for the nonuniform freestream conditions.

This study considered the MSL model with PD and RCS jets off. Future studies will focus on numerical simulations with these jets on to understand their aerodynamic interactions with the freestream and the aeroshell, in 
an effort to develop physically accurate and numerically efficient methods. The numerical results will also be assessed through qualitative and quantitative comparisons with experimental measurements.

The large continuum breakdown regions seen in the numerical results suggest that the Direct Simulation Monte Carlo (DSMC) method may be more appropriate in computing the flowfield properties in those regions. Future studies may also use a hybrid CFD-DSMC method where the continuum flow regions are simulated using CFD and the rarefied flow regions are simulated using DSMC. This hybrid method is more efficient than DSMC and more accurate than CFD.

\section{Acknowledgments}

The authors gratefully acknowledge funding for this work through NASA Grant NNX08AH37A. The use of supercomputers at the University of Michigan (NYX and BIGHOUSE clusters) and NASA (RTJones cluster) is essential to this work and is also greatly appreciated.

\section{References}

${ }^{1}$ Edquist, K. T., Hollis, B. R., Dyakonov, A. A., Laub, B., Wright, M. J., Rivellini, T. P., Slimko, E. M., and Willcockson, W. H., "Mars Science Laboratory Entry Capsule Aerothermodynamics and Thermal Protection System," IEEE Aerospace Conference Paper 2007-1423, March 2007.

${ }^{2}$ Korzun, A. M., Cruz, J. R., and Braun, R. D., "A Survey of Supersonic Retropropulsion Technology for Mars Entry, Descent, and Landing," IEEE Aerospace Conference Paper 2008-1246, March 2008.

${ }^{3}$ Cecil, E., and McDaniel, J. C., "Planar Velocity and Temperature Measurements in Rarefied Hypersonic Flow Using Iodine LIF," AIAA Paper 2005-4695, June 2005.

${ }^{4}$ McDaniel, J. C., Staack, D., Glass, C. E., and Miller, C., "Experimental and Computational Comparison of an Underexpanded Jet Flowfield," AIAA Paper 2002-0305, January 2002.

${ }^{5}$ Scalabrin, L. C., and Boyd, I. D., "Development of an Unstructured Navier-Stokes Solver for Hypersonic Nonequilibrium Aerothermodynamics", AIAA Paper 2005-5203, June 2005.

${ }^{6}$ Scalabrin, L. C., and Boyd, I. D., "Numerical Simulation of Weakly Ionized Hypersonic Flow for Reentry Configurations," AIAA Paper 2006-3773, June 2006.

${ }^{7}$ Holman, T. D., and Boyd, I. D., "Numerical Investigation of the Effects of Continuum Breakdown on Hypersonic Vehicle Surface Properties", AIAA Paper 2008-3928, June 2008.

${ }^{8}$ Karypis, G., and Kumar, V., "METIS: A Software Package for Partitioning Unstructured Graphs, Partitioning Meshes, and Computing Fill-Reducing Orderings of Sparse Matrices,” University of Minnesota, 1998.

${ }^{9}$ Wilke, C. R., “A Viscosity Equation for Gas Mixtures,” Journal of Chemical Physics, Vol. 18, 1950, pp. 517-519.

${ }^{10}$ Vincenti, W. G., and Kruger, C. H., Introduction to Physical Gas Dynamics, Krieger Publishing Company, 2002.

${ }^{11}$ Blottner, F. G., Johnson, M., and M. Ellis, "Chemically Reacting Viscous Flow Program for Multi-Component Gas Mixtures," Sandia Laboratories Report SC-RR-70-754, 1971.

${ }^{12}$ White, F. M., Viscous Fluid Flow, $3^{\text {rd }}$ ed., McGraw-Hill, 2006.

${ }^{13}$ Thermophysical Properties Database for Semiconductor Process Gases, National Institute of Standards and Technology.

${ }^{14}$ Padilla, J. F., "Assessment of Gas-Surface Interaction Models for Computation of Rarefied Hypersonic Flows," Ph.D. Thesis, Department of Aerospace Engineering, University of Michigan, Ann Arbor, MI, 2008.

${ }^{15}$ Ashkenas, H., and Sherman, F. S., "The Structure and Utilization of Supersonic Free Jets in Low Density Wind Tunnels," Contract No. NAS7-100, ONR/AFOSR Contract Review, 1965.

${ }^{16}$ GAMBIT 2.3 User's Guide. Fluent, 2009.

${ }^{17}$ Boyd, I. D., Chen, G., and Candler, G., "Predicting Failure of the Continuum Fluid Equations in Transitional Hypersonic Flows," Physics of Fluids, Vol. 7, 1995, pp. 210-219. 